\title{
WFPC2 STUDIES OF THE CRAB NEBULA. III. MAGNETIC RAYLEIGH-TAYLOR INSTABILITIES AND THE ORIGIN OF THE FILAMENTS ${ }^{1}$
}

\author{
J. Jeff Hester, ${ }^{2}$ James M. Stone, ${ }^{3}$ Paul A. Scowen, ${ }^{2}$ Byung-Il Jun, ${ }^{4}$ John S. Gallagher III, ${ }^{5}$ \\ Michael L. Norman, ${ }^{4}$ Gilda E. Ballester, ${ }^{6}$ Christopher J. Burrows, ${ }^{7}$ Stefano Casertano, ${ }^{7}$ \\ John T. Clarke, ${ }^{6}$ David Crisp,${ }^{8}$ Richard E. Griffiths, ${ }^{9}$ John G. Hoessel, ${ }^{5}$ \\ Jon A. Holtzman, ${ }^{8}$ John Krist, ${ }^{7}$ Jeremy R. Mould, ${ }^{10}$ Ravi Sankrit, ${ }^{2}$ \\ Karl R. Stapelfeldt, ${ }^{8}$ John T. Trauger, ${ }^{8}$ Alan Watson, ${ }^{11}$ \\ AND JAMES A. WESTPHAL 12 \\ Received 1995 April 3; accepted 1995 July 11
}

\begin{abstract}
Recently obtained Hubble Space Telescope WFPC2 images of the Crab Nebula show that the emission-line filaments are dominated by structures that morphologically appear to be the result of magnetic RayleighTaylor (R-T) instabilities at the interface between the pulsar-driven synchrotron nebula and a shell of sweptup ejecta. We replace this morphological argument with a quantitative treatment of the growth rate and characteristic wavelength of such instabilities. Using published data on the rate of expansion of the synchrotron nebula and the density of the ejecta, together with a wavelength for the instability measured from the WFPC2 images, we calculate a magnetic field strength of $\sim 540 \mu \mathrm{G}$. This is within a factor of 2 of the canonical minimum energy equipartition field of $300 \mu \mathrm{G}$, and probably closer than that to a more realistic estimate of the field at the edge of the Crab.

Comparison of the detailed morphology and ionization structure of the R-T fingers in the Crab with recent magnetohydrodynamical simulations which follow the development of magnetic R-T instabilities into the nonlinear regime is used to establish a sequence of filament properties which are determined by the density of the shell of swept-up ejecta at the edge of the synchrotron nebula. When the density is below a critical value, the interface is stable. For somewhat higher densities R-T instabilities grow, but the field, which becomes aligned along the length of the R-T fingers, is strong enough to prevent the development of secondary KelvinHelmholtz $(\mathrm{K}-\mathrm{H})$ instabilities as the finger falls through the lighter medium. At higher densities these $\mathrm{K}-\mathrm{H}$ instabilities develop, but the field is still strong enough to maintain a long streamer-like connection between the head of the filament and the shell. In a few cases, the density of the shell is high enough that the magnetic field is unable to prevent the fragmentation of R-T fingers, and the structure becomes more characteristic of a nonmagnetic R-T instability. The magnetic field is oriented along the length of an R-T finger, so material is free to "pour" into the finger from above. In equilibrium, gradients in thermal pressure and effective gravity must balance along field lines. As a result, loss of pressure support in the fingers due to cooling enhances the flow of material into the fingers, "siphoning" gas into the finger from above.

If an extended remnant of ejecta surrounds the visible extent of the Crab, as has been suggested frequently, then the synchrotron nebula is expanding through this extended remnant, sweeping up ejecta as it goes. R-T instabilities channel this swept-up ejecta into the hierarchy of dense visible filaments. It seems likely that the current system of filaments originated as a result of R-T instabilities as the synchrotron nebula expanded out through more uniformly distributed ejecta. If an extended remnant remains today, then filament formation is an ongoing process.

The ionization structure of filaments is also found to change in a systematic way as a function of the relative importance of the magnetic field and the mass density. Filaments which are dominated by the magnetic field are confined by the field and have sharp, well defined edges. Filaments in which the magnetic field is less dominant consist of high-density, low-ionization cores embedded within more extended high-ionization material. This confirms a previous suggestion that variations in magnetic confinement are an important caveat to published interpretations of spectra of Crab filaments.
\end{abstract}

Subject headings: instabilities - ISM: individual (Crab Nebula) - ISM : structure radiation mechanisms: nonthermal

\footnotetext{
${ }^{1}$ Based on observations with the NASA/ESA Hubble Space Telescope, obtained at the Space Telescope Science Institute, which is operated by AURA, Inc., under NASA contract 5-26555.

${ }^{2}$ Department of Physics and Astronomy, Box 871504, Arizona State University, Tempe, AZ 85287-1504.

${ }^{3}$ Department of Astronomy, University of Maryland, College Park, MD 20742-2421.

${ }^{4}$ Laboratory for Computational Astrophysics, National Center for Supercomputing Applications, Department of Astronomy, University of Illinois at Urbana-Champaign, 1002 West Green Street, Urbana, IL 61801.

5 Department of Astronomy, University of Wisconsin-Madison, 475 North Charter Street, Madison, WI 53706.
}

\footnotetext{
${ }^{6}$ Department of Atmospheric and Oceanic Sciences, University of Michigan, 2455 Hayward, Ann Arbor, MI 48109.

${ }^{7}$ Space Telescope Science Institute, 3700 San Martin Drive, Baltimore, MD 21218.

8 Jet Propulsion Laboratory, 4800 Oak Grove Drive, Pasadena, CA 91109.

${ }^{9}$ Department of Astronomy, Johns Hopkins University, 3400 North Charles Street, Baltimore, MD 21218.

${ }^{10}$ Lowell Observatory, Mars Hill Road, Flagstaff, AZ 86001.

11 Mount Stromlo and Siding Springs Observatories, Australian National University, Weston Creek Post Office, ACT 2611, Australia.

12 Division of Geological and Planetary Sciences, California Institute of Technology, Pasadena, CA 91125.
} 


\section{INTRODUCTION}

The interaction between the pulsar-driven Crab synchrotron nebula and the ejecta from the explosion is of long-standing interest. The importance of this interaction has been clear since observations by Trimble (1968) showed that the rate of expansion of the filaments is greater than expected for free expansion, implying that the filaments have been accelerated since the explosion.

Since the seminal paper by Rees \& Gunn (1974), a number of authors have considered the implications of the confinement of the Crab synchrotron nebula by its supernova remnant. These papers have often rested on a claim that most of the mass of the Crab resides in a "normal" remnant lying outside of the boundaries of the bright filaments (e.g., Chevalier 1977; Kennel \& Coroniti 1984a), which is responsible for confining the synchrotron nebula. Despite the fact that attempts to identify a more extended massive remnant remain inconclusive (e.g., Murdin 1995), the existence of such an extended remnant would help to answer many questions about the Crab. One thing that is clear from studies of the polarization of the Crab (e.g., Michel et al. 1991 and others) is that the magnetic field at the edge of the nebula "drapes over" the bright filaments, coupling the expansion of the synchrotron nebula as a whole to the cage of visible ejecta. It is also apparent in deep groundbased images and spectra of the Crab that the synchrotron nebula is bounded by a thin "skin" of high-ionization material (e.g., Clark et al. 1983).

It has been pointed out within the context of discussions of the confinement of the synchrotron nebula that the interface between the relativistic plasma and confining thermal material may be Rayleigh-Taylor (R-T) unstable (e.g., Chevalier \& Gull 1975; Bandiera, Pacini, \& Salvati 1983). Images of the Crab filaments obtained with the Hubble Space Telescope Wide Field and Planetary Camera 2 (WFPC2) provide compelling morphological evidence that this is the case (Hester et al. 1994, 1995b). Figure 1 (Plate 6) shows the sum of the WFPC2 [O III] and [O I] images over the full field of view of the instrument. The sum is used to allow both low- and highexcitation features to be seen in a single figure. The WFPC2 images show that the famous "filaments" seen in groundbased images themselves consist of a relatively faint, highionization outer skin, from which multiple lower ionization "fingers" protrude into the interior of the synchrotron bubble. This morphology lead Hester et al. $(1994,1995 b)$ to suggest that the current structure of the Crab filaments are the result of magnetic R-T instabilities at the interface between the synchrotron nebula and an outer skin of swept-up ejecta which bounds the synchrotron bubble.

In the present paper we undertake a more quantitative discussion of magnetic $\mathrm{R}-\mathrm{T}$ instabilities in the Crab. In $\S 2$ we describe the interface at the boundary of the synchrotron nebula and use a linear growth rate analysis (Chandrasekhar 1961) to establish that this interface is unstable. Recently, Jun, Norman, \& Stone (1995, hereafter JNS95) have presented twoand three-dimensional numerical simulations of the development of magnetic R-T instabilities for a variety of field strengths and directions with respect to the interface. These authors find that in the nonlinear regime, the morphology and structure of the fingers produced by the instability are a strong function of the magnetic field strength. For strong fields, smallscale structure is suppressed, resulting in long, smooth fingers which are strikingly similar to those observed in the WFPC2 images. In $\S 3$ we apply these numerical results to the $\mathrm{Crab}$ and find that there is a well defined progression of filament morphologies which can be understood in terms of the ratio of the density at the interface to a critical density below which the interface is stabilized by the field. Caveats such as the effect of cooling are discussed in \$3.4. The implications of our findings for the overall structure of the Crab and the ionization structure of the filaments are discussed in $\S 4$. Section 5 summarizes the work.

\section{THE R-T UNSTABLE INTERFACE AT THE BOUNDARY OF THE SYNCHROTRON NEBULA}

\subsection{The "Skin" at the Boundary of the Nebula}

We begin by describing the interface that appears to exist at the boundary of the Crab Nebula. The WFPC2 images (Fig. 1) contain multiple fingers of emission protruding into the interior of the synchrotron nebula. These "radial" fingers are joined at their tops by faint, thin, "tangential," and often somewhat arcuate features which trace the location of the R-T unstable interface, itself. A schematic diagram of the situation is shown in Figure 2. The edge of the synchrotron nebula, which is moving outward at greater than the local free expansion velocity, has swept up what appears from the data to be a relatively thin "skin" of ejecta. This material is being accelerated by the pressure of the synchrotron nebula, giving rise to a local "effective gravity" pointing back into the interior of the nebula. If the buoyancy forces are greater than the magnetic tension at this interface, then it will be R-T unstable, as shown.

In order for the observed R-T instabilities to develop, there must be an appreciable drop in pressure across the band of emission connecting the top of each R-T finger to its neighbors. It is possible that these connecting features define locations of pressure discontinuities within the synchrotron nebula. However, it seems more likely that the features instead lie on the local boundary of the synchrotron nebula. Brief inspection of the WFPC2 images may not, in itself, demand this conclusion. The physical relationship between the connecting features and the synchrotron nebula is confused by the fact that they are seen in projection against the body of the synchrotron nebula. However, other data, together with a more careful consideration of the WFPC2 images, supports this association.

Deep, ground-based images of the Crab in high-ionization lines such as [O III] show that the synchrotron nebula is almost entirely bounded by faint, high-ionization emission. It seems very likely that this faint outer boundary of emission is the same as the faint high-velocity emission seen across the front and back faces of the nebula as well (Clark et al. 1983). Observationally, this emission seems to very nearly cover the surface of the synchrotron nebula. Moving to the WFPC2 images, the emission connecting adjacent fingers forms almost unbroken structures over very large scales (e.g., the loop of emission spanning the WNW quadrant of the field and the band of emission running NW to SE across the NNE quadrant of the field). The contiguous appearance of this emission makes it unlikely that it consists of fragments which have been "engulfed" by the synchrotron nebula. Rather, it seems more likely that the emission connecting the R-T fingers in the WFPC2 images is part of the same high-ionization "skin" seen in ground-based data. This is further supported by the isovelocity images of the Crab published by Lawrence et al. (1995), which show that while the Crab filaments may penetrate deep into the interior of the nebula, they are almost 


\section{PLATE 6}

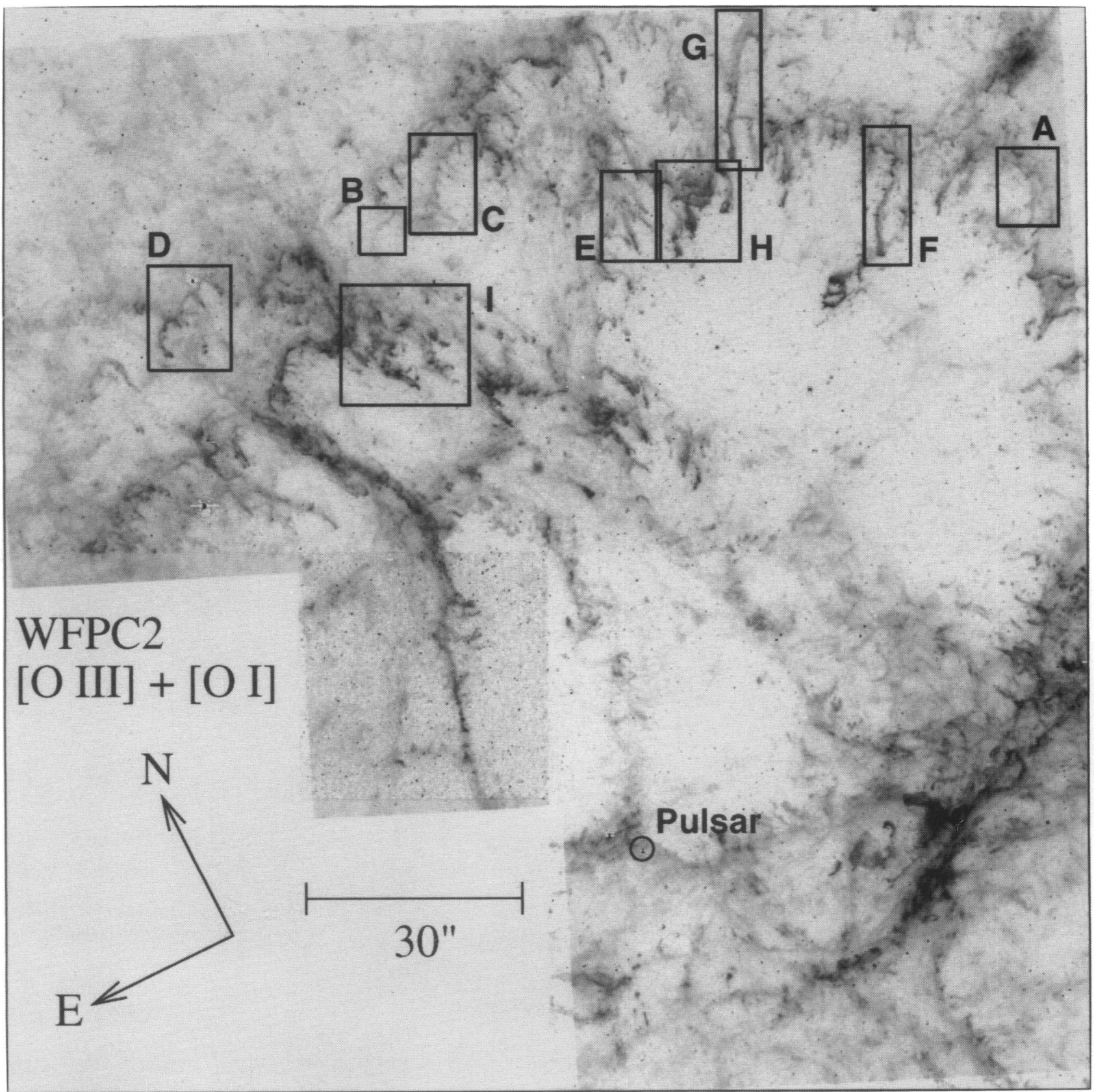

FIG. 1.-A weighted sum of the WFPC2 [O III] and [O $\mathrm{I}]$ images of a field in the NW part of the Crab Nebula. The full field of view of the WFPC2 is shown. The small boxes labeled A-I show regions which are selected as examples of different filament morphologies. The small circle indicates the location of the pulsar. Directions on the sky are indicated. The bar has a length of $30^{\prime \prime}$, which corresponds to $9 \times 10^{17} \mathrm{~cm}$ at an assumed distance of $2 \mathrm{kpc}$.

Hester et al. (see 456, 226) 
(Extended region

of ejecta?)

\section{Acceleration due to pressure of synchrotron nebula}

Projection of $\mathrm{g}$ along interface and siphon effect cause material

to "drain" into fingers.

\section{"Skin" of ejecta at boundary}

of synchrotron nebula

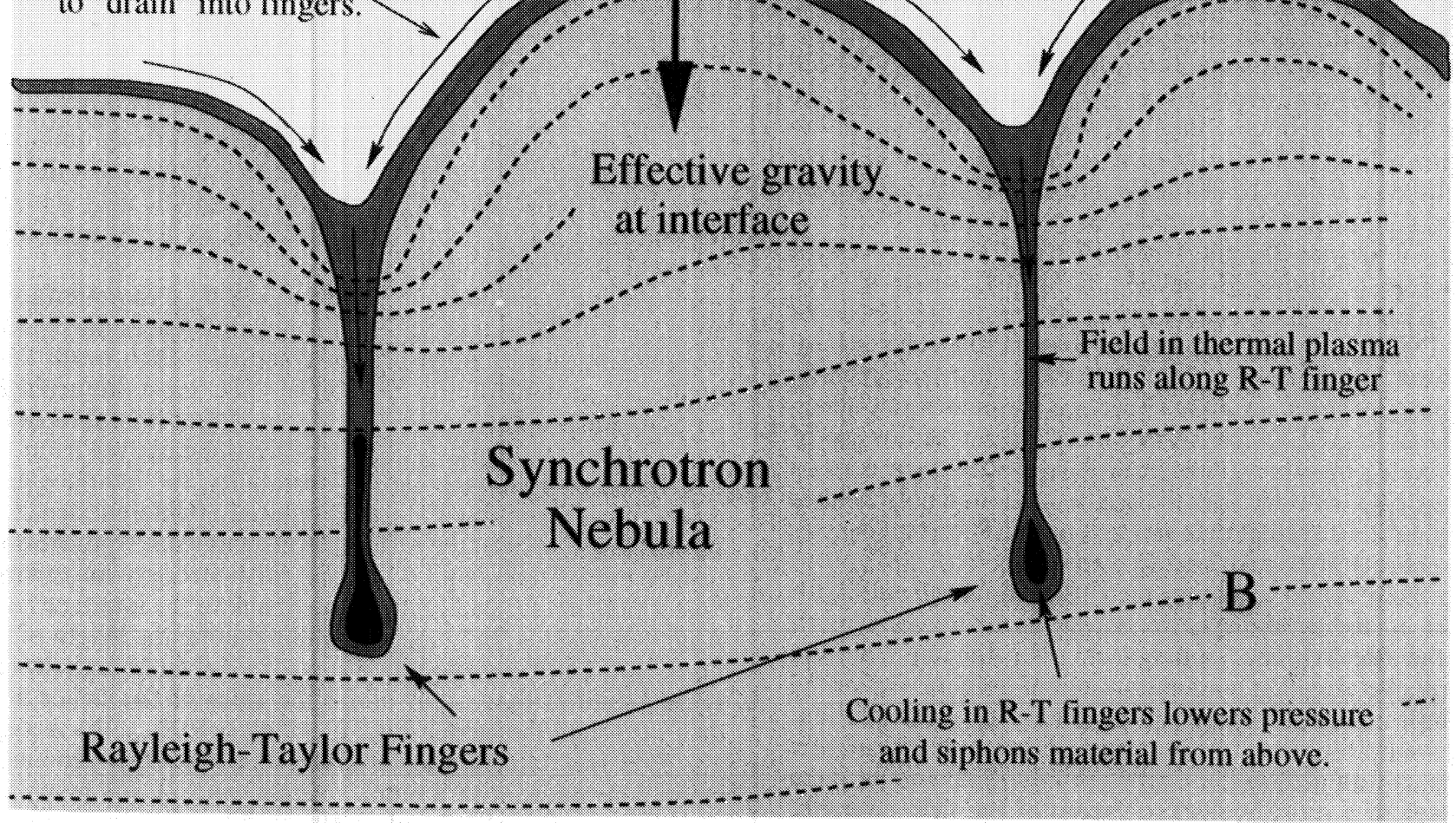

FIG. 2.-A sketch showing the structure of the R-T unstable interface at the boundary of the Crab synchrotron nebula. The pressure of the confined relativistic plasma accelerates a swept-up "skin" of thermal ejecta, resulting in an effective gravity directed into the interior of the remnant. If magnetic tension is too weak to stabilize the interface, R-T modes grow. Note that field lines in the synchrotron nebula do not actually interpenetrate the filaments as shown, but rather drape around them in the third dimension. The magnetic field within the R-T fingers is aligned with the finger, allowing thermal plasma from the skin to readily flow into the finger.

always connected back to the outer surface of the Crab, making them topologically part of this surface.

Throughout this paper we use the term "skin" to refer to this thin shell of thermal material which marks the local boundary of the synchrotron nebula. It is the "skin" of the Crab which we infer below to be R-T unstable.

\subsection{Application of Linear $R-T$ Theory to the Crab}

A linear analysis of the growth rates of magnetic R-T instabilities was presented by Chandrasekhar (1961), and was recently reviewed by JNS95. In a nonmagnetic RayleighTaylor instability, the growth rate increases with decreasing wavelength. However, for a given density contrast, effective gravity, and magnetic field, there is a critical wavelength below which the instability is stabilized by magnetic tension. The wavelength which grows fastest, and hence should dominate the structure of an unstable interface, is twice this critical value, or

$$
\lambda_{u}=\frac{2 B^{2} \cos ^{2} \theta}{g\left(\rho_{2}-\rho_{1}\right)},
$$

where $B$ is magnetic field, $\theta$ is the angle between the field and the surface of the interface, $g$ is the effective gravity, and $\rho_{1}$ and $\rho_{2}$ are the densities of the light and heavy fluids, respectively.

To compare the theory of magnetic R-T instabilities with the WFPC2 observations of the Crab, we begin by estimating the wavelength of the observed instability. This is complicated by possible line of sight confusion, which is clearly present in most locations within the field of view, and which causes fingers to appear to be closer together than they actually are. We looked through the data and chose a number of locations where the geometry appeared simple, with several regularly spaced fingers which were joined smoothly to their neighbors (e.g., regions $\mathrm{C}$ and $\mathrm{D}$ below). It seems unlikely that fingers spaced widely along the line of sight would look so regular or would appear to form such a well defined and interconnected structure. These are also regions which are not far beyond the linear regime of the instability, so the expression above should be applicable. After applying a statistical correction of $2^{1 / 2}$ to account for line of sight effects that may make the observed separation smaller than the actual wavelength, we estimate that the wavelength of the instability is $1^{\prime \prime} .5 \lesssim \lambda_{\text {obs }} \lesssim 5^{\prime \prime}$, with a representative value of $\sim 3^{\prime \prime}$.

The acceleration, density, and magnetic field in the Crab can all be estimated on the basis of published data. The acceleration of the Crab filaments was originally noted by Trimble (1968), who found that the current rate of expansion of the filaments extrapolates back to an origin in the year A.D. 1140, 86 years after the known explosion date in A.D. 1054. Similarly, Wyckoff \& Murray (1977) found an expansion date of A.D. 1120. However, these observations are dominated by the bright filaments, which protrude into the interior of the Crab (i.e., the dense material which has "fallen into" the Crab as a result of the instability) and should have undergone less acceleration 
than material on the surface of the synchrotron bubble. A more relevant value comes from Bietenholz et al. (1991), who measured the rate of expansion of the outer edge of the radio synchrotron nebula and determined a significantly later expansion date of A.D. $1257 \pm 22$ years. A present-day measurement of expansion velocity does not unambiguously determine the history of acceleration of the Crab filaments. If we make the minimal assumption (the details of which do not strongly affect our conclusions) that acceleration has been roughly constant over the life of the remnant, then the observed expansion of the edge of the nebula gives a value of $g=3.53 \times 10^{-3} \mathrm{~cm} \mathrm{~s}^{-2}$.

The electron density of filaments in the Crab has been measured by a number of authors. Fesen \& Kirshner (1982), for example, found that $550 \mathrm{~cm}^{-3} \leq n_{e}([\mathrm{~S}$ II $]) \leq 1700 \mathrm{~cm}^{3}$ for a sample of 11 positions. However, the WFPC2 images show that [S II] emission is concentrated in the dense filaments which have "fallen into" the synchrotron nebula. The density of the swept-up ejecta lying on the outer skin of the synchrotron bubble is almost certainly lower; we adopt as a characteristic value $275 \mathrm{~cm}^{-3}$, which is a factor of 2 below the low end of the observed range of values. This is converted into a mass density $\rho_{2}$ by assuming that the gas is predominately helium (MacAlpine et al. 1989) and is singly ionized. The mass density of the synchrotron plasma is set to zero.

Using these values, equating the observed wavelength with the most unstable and hence fastest growing wavelength during the linear phase, and ignoring for the moment the $\cos \theta$ term, we calculate a magnetic field of $540 \mu \mathrm{G}$. Given the uncertainties in our estimates of the density and acceleration, this is remarkably close to the canonical value of $300 \mu \mathrm{G}$ for the equipartition field in the Crab (Trimble 1982) and perhaps even closer to a more realistic estimate of the field strength. Hester et al. (1995a) found that small-scale brightness variations within the synchrotron nebula probably require that the field be higher than the minimum energy value by a factor of $\sim 2$. Further, theoretical calculations of the structure of the synchrotron nebula (e.g., Kennel \& Coroniti 1984a) predict that the pressure should reside predominately in the magnetic field at the edge of the nebula.

The values used in these calculations are somewhat uncertain and vary within the nebula. In the sections to follow, for example, we will consider the effects of variations in the density of the thermal skin on how the instability develops. It is important to emphasize, however, that these uncertainties do not affect the conclusion that the skin of the Crab Nebula is in the regime where magnetic $\mathrm{R}-\mathrm{T}$ instabilities between the synchrotron nebula and thermal material will occur. There are no strong dependencies in the expression from the linear theory relating $\lambda, g, \rho$, and $B$-the magnetic field is proportional to only the $\frac{1}{2}$ power of the other three quantities-and no "tuning" of parameters was required to make the theory fit. This relationship is robust to reasonable variations in estimates of these four quantities. We conclude that the magnetic $\mathrm{R}-\mathrm{T}$ instabilities in the Crab, originally identified on the basis of morphology, are quantitatively accounted for by the linear theory.

\section{COMPARISON WITH MHD SIMULATIONS OF MAGNETIC R-T INSTABILITIES \\ 3.1. Summary of the Results of Numerical Calculations}

Having quantitatively accounted for the presence of magnetic R-T instabilities in the Crab, we now use the results of the numerical models to investigate the physical nature of the observed structure of the filaments. The calculations of JNS95 show that the development of the instability is a function of the strength of the magnetic field in comparison to the critical field strength (Chandrasekhar 1961) given by

$$
B_{\text {crit }}=\frac{\left[g \lambda\left(\rho_{2}-\rho_{1}\right)\right]^{1 / 2}}{\cos \theta}
$$

If $B>B_{\text {crit }}$, then the buoyancy forces are relatively unimportant compared to field tension, and the interface is stable. In the other extreme, if $B \ll B_{\text {crit, }}$, then the magnetic field is unimportant, and the instability behaves just as a classical nonmagnetic R-T instability.

In the intermediate regime, where $B \leqslant B_{\text {crit }}$, the interface is unstable, but the magnetic field strongly influences the characteristics and morphology of the structures which result from nonlinear growth of the instability. The most immediately obvious effect of the field in this regime is that it inhibits dense knots of material from totally detaching themselves from the interface. In a nonmagnetic R-T instability, the interface fragments into disconnected blobs of material of different densities. In the magnetic case, the instability instead leads to the formation of long, "tadpole-like" fingers, with strongly magnetized tails which tie the head of the feature back to the interface itself. $A$ related effect is that the field running along the length of the finger may be strong enough to suppress Kelvin-Helmholtz (K$\mathrm{H}$ ) instabilities that occur along the sides of the finger as it drops through the lighter fluid. If $B$ is close to $B_{\text {crie }}$, the secondary $\mathrm{K} \cdot \mathrm{H}$ instabilities are stabilized, and the finger remains a smooth structure with a blunt, rounded end. If $B$ is much less than $B_{\text {crit }}$, on the other hand, K-H instabilities grow, leading to the development of a flattened or anvil-like head, and the presence of vortices and other structures associated with K-H instabilities.

These effects can be seen in Figure 3, which shows numerical calculations of the growth of a multimode instability (JNS95). Figure $3 a$ shows the nonmagnetic case. Numerous K-H swirls are apparent, and the $\mathrm{R}-\mathrm{T}$ fingers are being shredded. The case with a tangential field $(\cos \theta=1)$ of $0.2 B_{\text {crit }}$ is shown in Figure $3 b$. The magnetic field prevents the development of K-H instabilities, leading to the formation of long, well developed fingers connected by smooth bubbles. Also note that in the nonmagnetic case the dense fingers are embedded within a more extended region of intermediate density material. In the magnetic case, on the other hand, the fingers have sharply defined boundaries and the filling factor for intermediate density material is very low.

\subsection{Behavior of the Instability as a Function of Density}

It is likely that the magnetic field at the surface of the synchrotron bubble is relatively constant throughout the outer regions of the Crab. This is because the extremely high sound speed in the interior of the nebula does not allow large pressure differences to develop and because the pressure resides mainly in the field at the edge of the nebula. (This is not strictly true; the field strength should be somewhat higher at locations where the field is draped over dense filaments.) Using the parameters above, and assuming a temperature of $10^{4} \mathrm{~K}$, the thermal pressure in the filaments at the edge of the Crab is over an order of magnitude smaller than the magnetic pressure. 


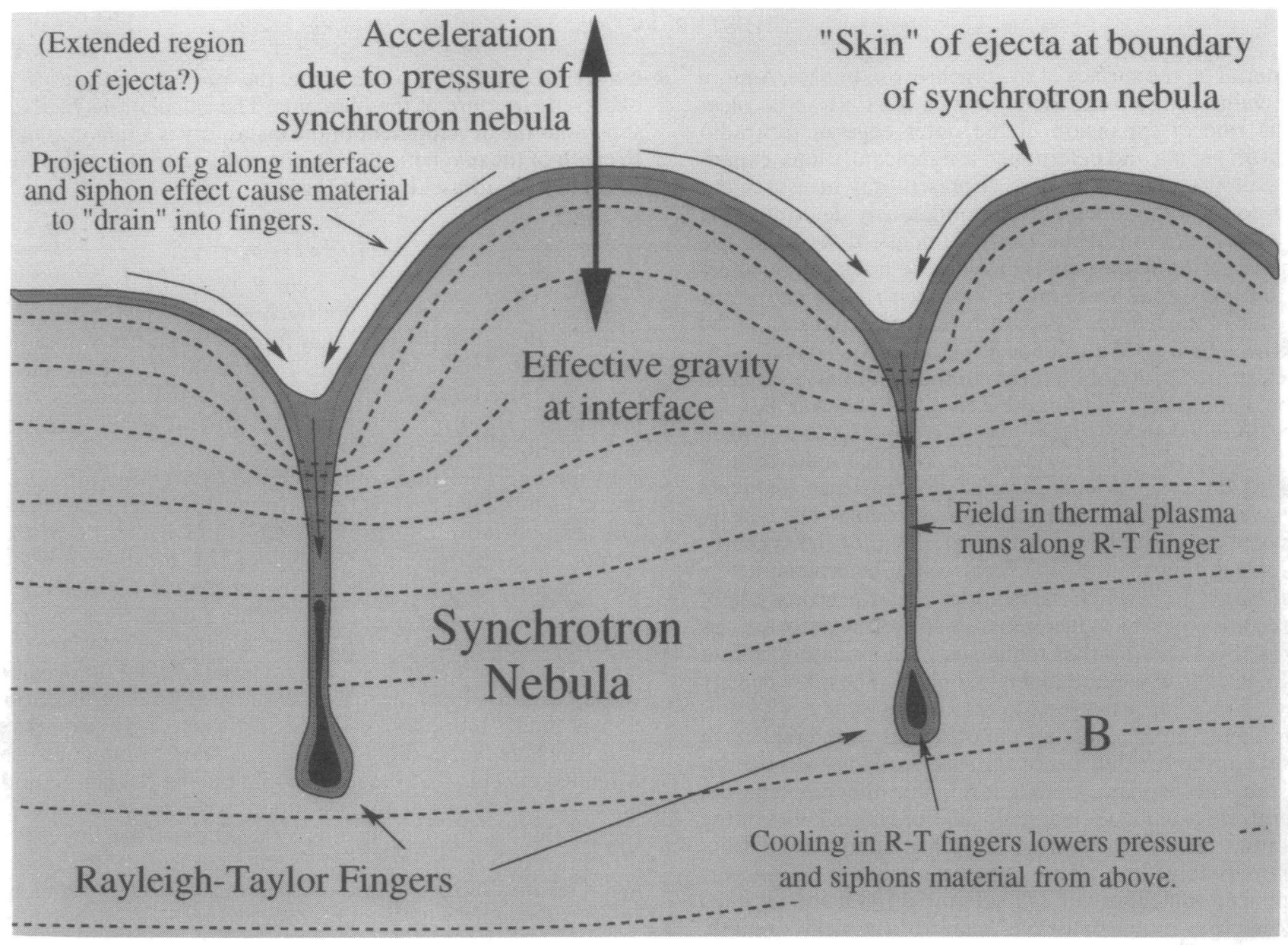

FIG. 2.-A sketch showing the structure of the R-T unstable interface at the boundary of the Crab synchrotron nebula. The pressure of the confined relativistic plasma accelerates a swept-up "skin" of thermal ejecta, resulting in an effective gravity directed into the interior of the remnant. If magnetic tension is too weak to stabilize the interface, R-T modes grow. Note that field lines in the synchrotron nebula do not actually interpenetrate the filaments as shown, but rather drape around them in the third dimension. The magnetic field within the R-T fingers is aligned with the finger, allowing thermal plasma from the skin to readily flow into the finger.

always connected back to the outer surface of the Crab, making them topologically part of this surface.

Throughout this paper we use the term "skin" to refer to this thin shell of thermal material which marks the local boundary of the synchrotron nebula. It is the "skin" of the Crab which we infer below to be R-T unstable.

\subsection{Application of Linear $R-T$ Theory to the Crab}

A linear analysis of the growth rates of magnetic R-T instabilities was presented by Chandrasekhar (1961), and was recently reviewed by JNS95. In a nonmagnetic RayleighTaylor instability, the growth rate increases with decreasing wavelength. However, for a given density contrast, effective gravity, and magnetic field, there is a critical wavelength below which the instability is stabilized by magnetic tension. The wavelength which grows fastest, and hence should dominate the structure of an unstable interface, is twice this critical value, or

$$
\lambda_{u}=\frac{2 B^{2} \cos ^{2} \theta}{g\left(\rho_{2}-\rho_{1}\right)},
$$

where $B$ is magnetic field, $\theta$ is the angle between the field and the surface of the interface, $g$ is the effective gravity, and $\rho_{1}$ and $\rho_{2}$ are the densities of the light and heavy fluids, respectively.

To compare the theory of magnetic R-T instabilities with the WFPC2 observations of the Crab, we begin by estimating the wavelength of the observed instability. This is complicated by possible line of sight confusion, which is clearly present in most locations within the field of view, and which causes fingers to appear to be closer together than they actually are. We looked through the data and chose a number of locations where the geometry appeared simple, with several regularly spaced fingers which were joined smoothly to their neighbors (e.g., regions $C$ and $D$ below). It seems unlikely that fingers spaced widely along the line of sight would look so regular or would appear to form such a well defined and interconnected structure. These are also regions which are not far beyond the linear regime of the instability, so the expression above should be applicable. After applying a statistical correction of $2^{1 / 2}$ to account for line of sight effects that may make the observed separation smaller than the actual wavelength, we estimate that the wavelength of the instability is $1^{\prime \prime} .5 \lesssim \lambda_{\text {obs }} \lesssim 5^{\prime \prime}$, with a representative value of $\sim 3^{\prime \prime}$.

The acceleration, density, and magnetic field in the Crab can all be estimated on the basis of published data. The acceleration of the Crab filaments was originally noted by Trimble (1968), who found that the current rate of expansion of the filaments extrapolates back to an origin in the year A.D. 1140, 86 years after the known explosion date in A.D. 1054. Similarly, Wyckoff \& Murray (1977) found an expansion date of A.D. 1120. However, these observations are dominated by the bright filaments, which protrude into the interior of the Crab (i.e., the dense material which has "fallen into" the Crab as a result of the instability) and should have undergone less acceleration 

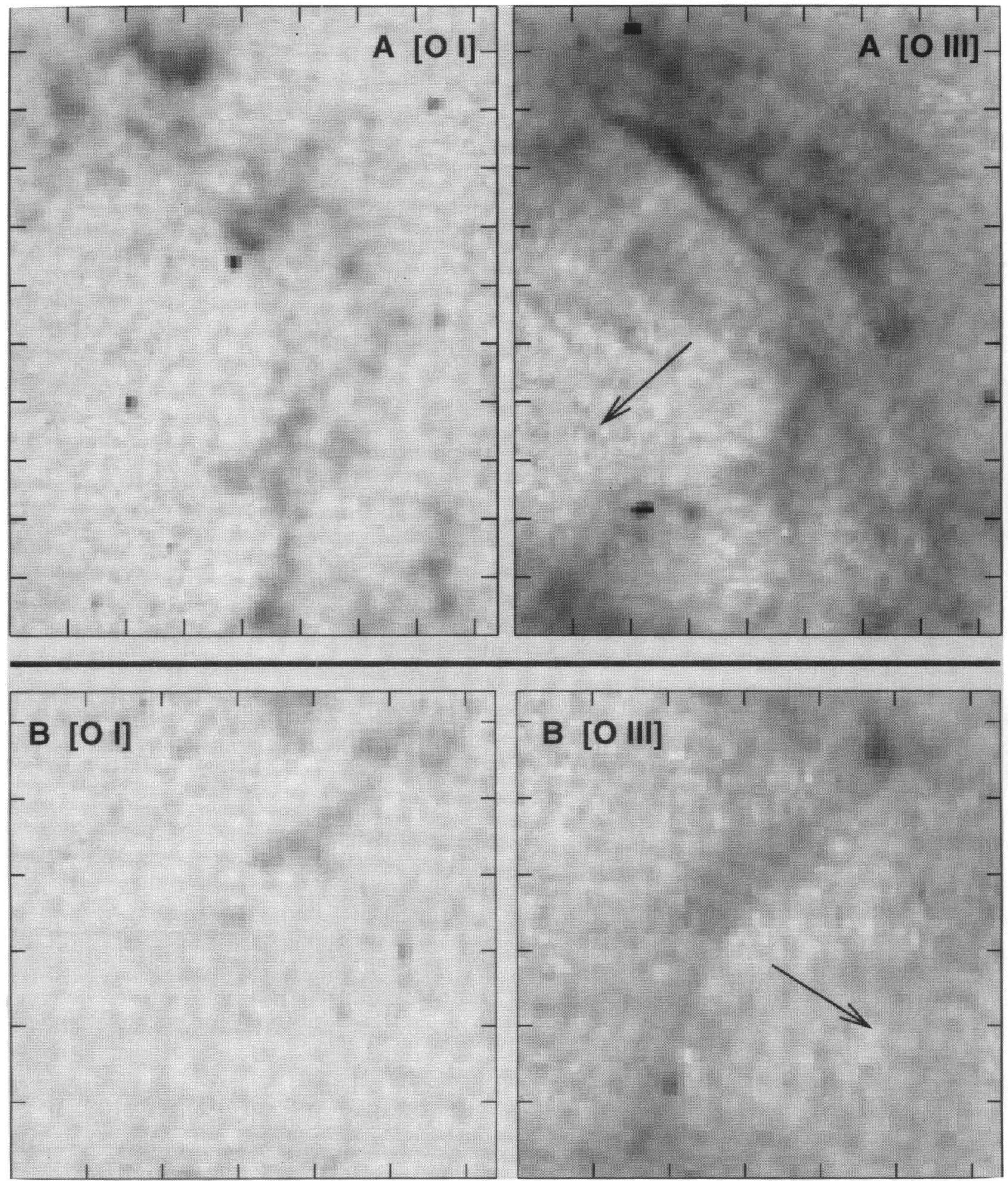

Fig. 4.-Enlargements of the WFPC2 [O I] $\lambda 6300$ and [O III] $\lambda 5007$ images for regions A and B. These are examples of locations in the Crab Nebula where the density of the swept-up ejecta at the outer boundary of the synchrotron nebula is low enough $\left(\rho / \rho_{\text {crit }}<1\right)$ that the magnetic field stabilizes the interface. Each tick mark on the side of the figures is $1^{\prime \prime}$ long, corresponding to $3 \times 10^{16} \mathrm{~cm}$. The small arrows in the [O III] images show the local normal to the interface, which is the direction of the effective gravity. In Figs. 4-7 a square root stretch is used to improve the dynamic range of the display. All of the images in a given line are displayed using the same stretch across Figs. 4-7, and so can be directly compared with each other.

HeSTER et al. (see 456,229$)$ 
PLATE 8

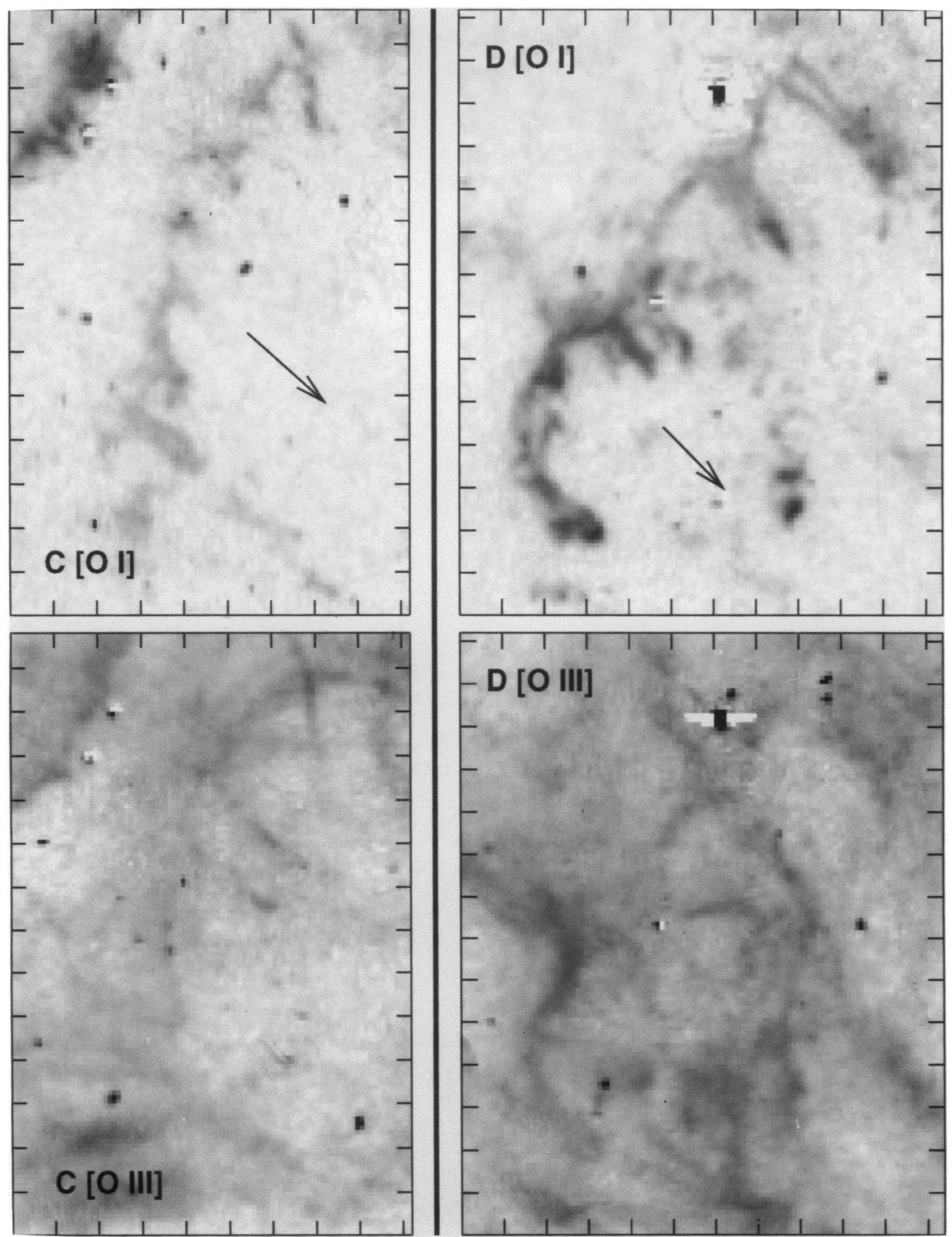

FIG. 5.-Enlargements of the WFPC2 [O III] and [O III] images of regions $\mathrm{C}$ and D. In these regions the interface between the synchrotron nebula and the skin of swept-up ejecta is marginally unstable $\left(\rho / \rho_{\text {crit }} \gtrsim 1\right)$. The difference in density and ionization state between the R-T fingers (which have low ionization and hence higher density) and the skin at the surface of the nebula (which has higher ionization and hence lower density) is apparent.

HESTER et al. (see 456, 229) 

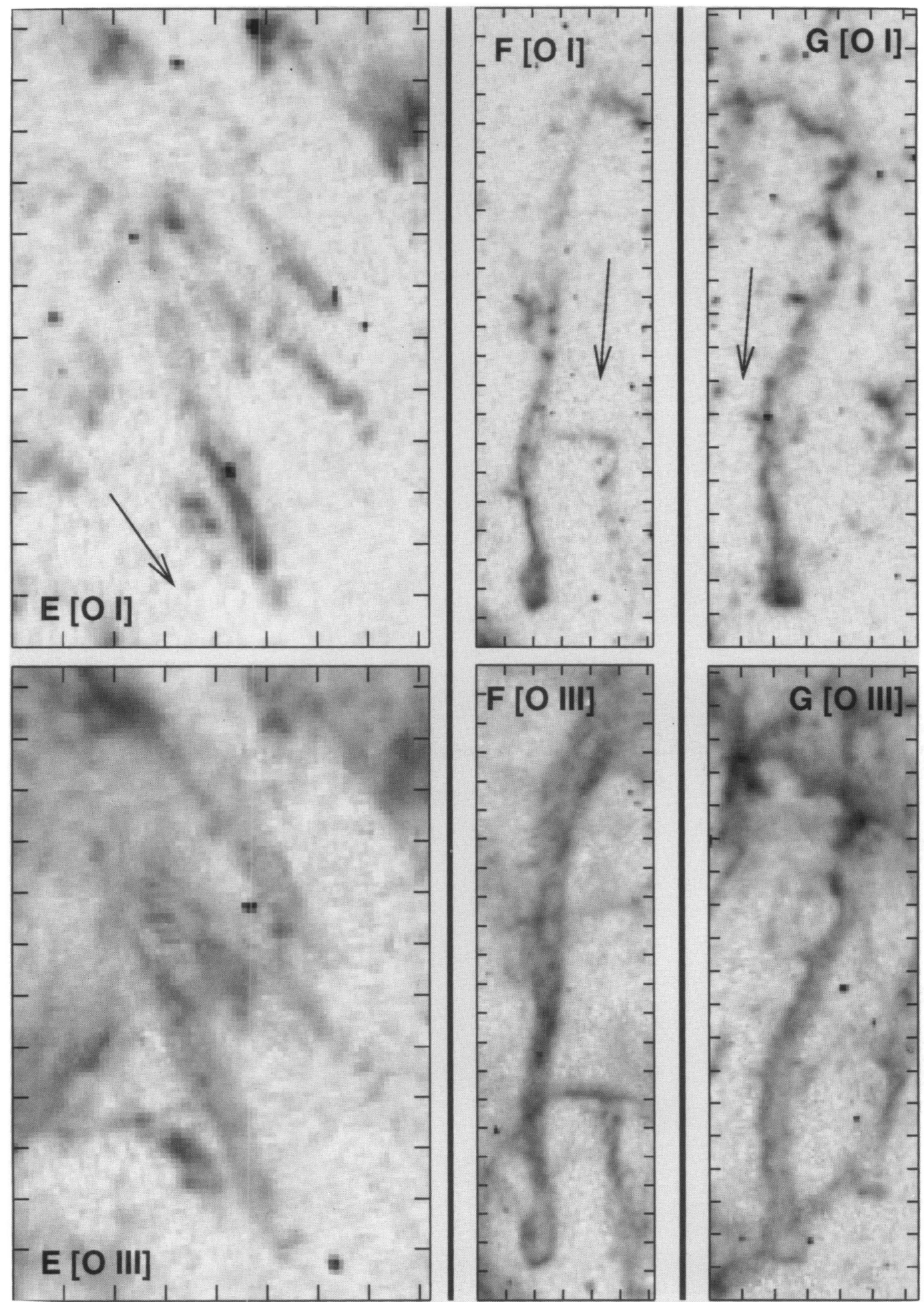

Fig. 6.-Enlargements of the WFPC2 [O I] and [O III] images of regions E, F, and G. This sequence documents the development of secondary $\mathrm{K}-\mathrm{H}$ instabilities in the R-T fingers. In region $E$ (as in regions $C$ and D) the interface is R-T unstable, but the field is strong enough to suppress the development of K-H instabilities at the head and along the edges of the finger. The R-T fingers have rounded tips. The filament in region $\mathrm{F}$ is beginning to show some sign of a more bulbous head, but the edges of the filament are still relatively smooth. In region G, the density is high enough that the field is no longer able to completely stabilize the K-H instabilities that occur as the R-T finger falls through the lighter material. The feature has a well defined "mushroom cap" head and shows other structure characteristic of K-H instabilities along its length.

Hester et al. (see 456, 229) 
!':

PLATE 10

I!
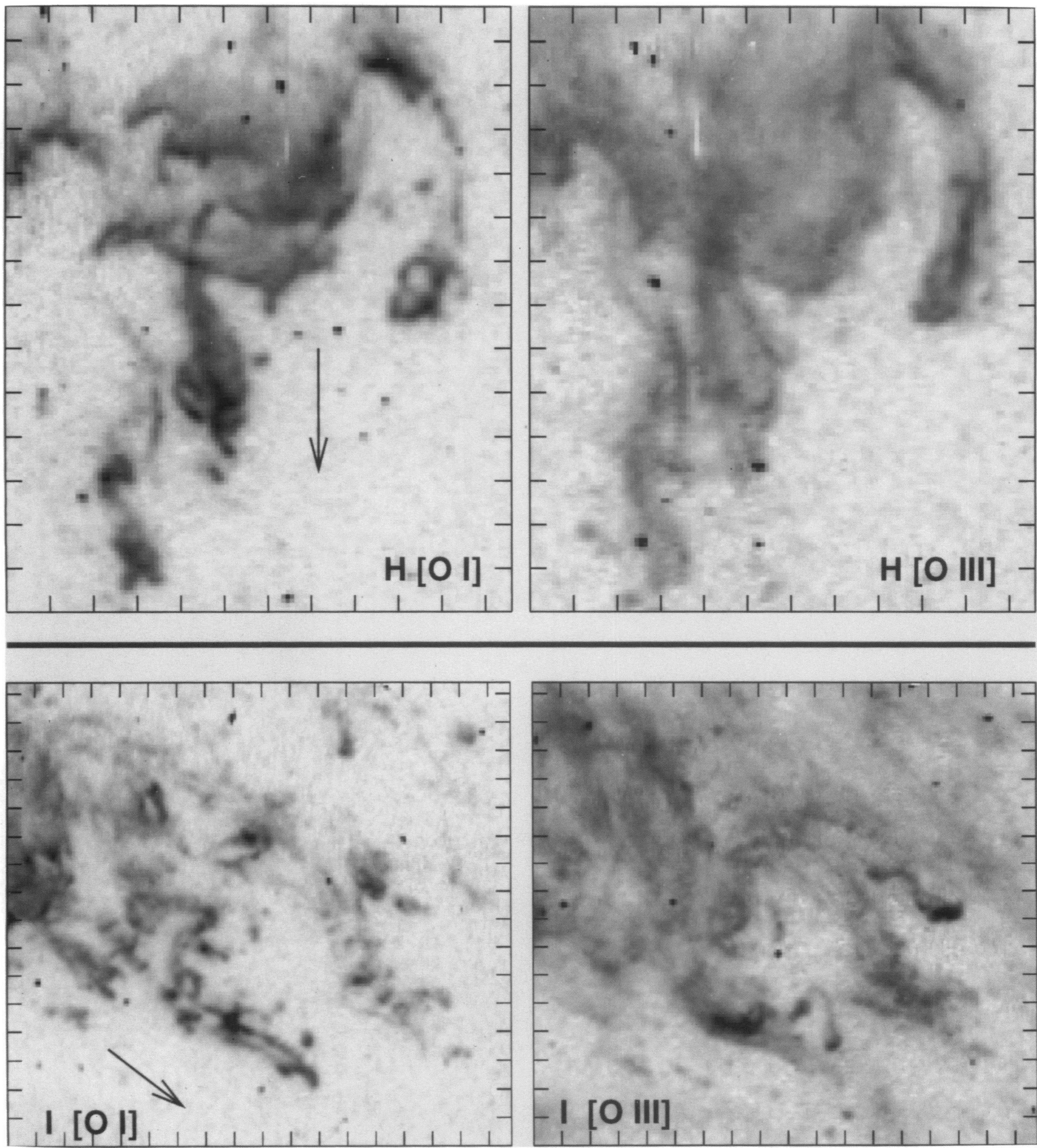

FIG. 7.-Enlargements of the WFPC2 [O I $]$ and [O III] images of regions $\mathrm{H}$ and $\mathrm{I}$. These two regions are very bright, especially in the low ionization [O $\mathrm{I}]$ line. The density here is high enough that the magnetic field is ineffective in stabilizing secondary K-H instabilities. In region I especially, the R-T fingers have largely fragmented, as happens in the nonmagnetic case.

Hester et al. (see 456,229$)$ 


\subsection{2. $\rho / \rho_{\text {crit }}>1$ : Appearance of Secondary $K-H$ Instabilities}

Figure 6 shows regions E, F, and G. Region E contains several R-T fingers which have generally grown longer than the structures in regions $C$ and $D$. Like the structures in regions $C$ and $D$, these fingers have blunt ends and show little evidence for $\mathrm{K}-\mathrm{H}$ instabilities along their edges. As with regions $\mathrm{C}$ and $\mathrm{D}$, the low-ionization emission is concentrated in the heads of the structures. A new feature of these fingers is low-ionization cores surrounded by higher ionization envelopes.

The trend toward higher density at the interface continues through regions $F$ and $G$, which contain very long $R-T$ fingers with pronounced head-tail and core-envelope structures. (The "hole" in the center of the [O $\mathrm{I}]$ emission at the head of the filament in F is not a hole, but instead an even higher density, cooler core including dust which is seen in absorption against the synchrotron background [Hester et al. 1994, 1995b].) The ionization of the R-T finger in region $G$ shows an even better defined core-halo structure. The effects of $\mathrm{K}-\mathrm{H}$ instabilities are also becoming apparent along the length of the finger in region $\mathrm{G}$, and in the characteristic "mushroom" cap at the head of the feature. The progression in $\rho / \rho_{\text {crit }}$ across regions $E, F$, and $G$ in Figure 6 spans the threshold for magnetic stabilization of $\mathrm{K}-\mathrm{H}$ instabilities in the falling finger.

\subsection{3. $\rho / \rho_{\text {crit }} \gg 1:$ Nearly Nonmagnetic Instabilities}

The final regions in the sequence are presented in Figure 7. Region $\mathrm{H}$ is very bright and contains a number of fingers with complex substructure, including multiple loops and swirls and other features which give a clear indication that the fingers are $\mathrm{K}-\mathrm{H}$ unstable. This structure is most pronounced in [O $\mathrm{I}$ ]. Emission from [O III] is again more diffuse and surrounds the [O I] features. The final region in the sequence is region I. Here the R-T fingers fragment into many isolated low-ionization knots, with very little head-tail structure remaining. These knots are embedded within a region of bright diffuse highionization emission. The structure is much like that resulting from a nonmagnetic R-T instability. The interface density at this location was very high, and the magnetic field was relatively unimportant in the development of the instability.

The trend discussed in the preceding sections is clearly a trend (1) toward increasing density (on the basis of brightness and ionization structure) and (2) toward increasing importance of $\mathrm{K}-\mathrm{H}$ instabilities. In a purely nonmagnetic instability, on the other hand, $\mathrm{K}-\mathrm{H}$ instabilities become less pronounced for larger density differentials (e.g., Gardner et al. 1988). This is further evidence that the relative importance of the magnetic field is of paramount importance in determining the structure of the Crab filaments.

\subsubsection{Hierarchical Structure}

The "filaments" seen in the HST images of the Crab are not the same as the structures normally referred to as "filaments." Rather, the "filaments" which dominate ground-based images (e.g., Fig. 1 in Lawrence et al. 1995) appear in the WFPC2 images (Fig. 1) as broad bands of emission. The R-T fingers discussed in this paper are substructures within the major filaments, which in turn form the backbone of the overall filamentary cage. There is clearly a hierarchy to the filamentary structure of the Crab. This hierarchy is depicted schematically in Figure 8. (The WFPC2 images discussed here document the small-scale structures; Fig. 8 should also be compared with the zero velocity cross section of the Crab from Lawrence et al.
1995 to see the larger scale features.) This is as expected from the development of a multimode R-T instability, where in the nonlinear regime the longest wavelengths grow fastest. An interesting question is whether this instability alone will be able to explain the "strands of spaghetti on a balloon" morphology (Davidson \& Fesen 1985) exhibited by the "filaments" noted from the ground.

\subsection{Effect of Cooling}

There are a number of caveats to the discussion above. For example, the simulations assume $\gamma=1.4$ for both fluids, while in the Crab the dense fluid has $\gamma=5 / 3$ and the light fluid has $\gamma=4 / 3$. The details of the secondary $\mathrm{K}-\mathrm{H}$ instabilities between a thermal plasma and a relativistic plasma with crossed magnetic fields deserves better attention as well. The most significant shortcoming of the numerical simulations, however, is that they neglect cooling.

In the Crab, material collects in the dense cores of the R-T fingers, where it is shielded from ionizing synchrotron radiation and is able to cool and recombine. This is clear from the low-ionization emission, dust (Hester et al. 1994, 1995b), and molecular emission (Graham et al. 1990) concentrated there. Since there is no magnetic support along the field lines running along the length of the fingers, the sum of the component of the effective gravity and the pressure gradient along field lines must balance in a steady state. However, cooling can lower the thermal pressure in the dense tips of the fingers substantially, resulting in both unbalanced gravitational forces and a net pressure gradient which siphons material into the fingers and toward their tips. A similar effect is familiar in time lapse photography of flux tubes associated with solar prominences (see, e.g., the review by Low 1990). As fluid pours into the fingers from above, the density at the tip will be strongly enhanced.

Comparison with simulations suggests that $\rho / \rho_{\text {crit }} \sim 1.5$ in the marginally unstable structures in regions $C$ and $D$ (Fig. 5). Taking $n_{\text {crit }} \approx 170$ atoms $\mathrm{cm}^{-3}$, the density at the interface which leads to the structures in regions $C$ and D was probably $\sim 250$ atoms $\mathrm{cm}^{-3}$. The present density in these fingers is much higher than this. A WFPC2 image taken in the light of He I $\lambda 5876$ was used to estimate a volume emissivity of $\sim 1.4 \times 10^{-20} \mathrm{ergs} \mathrm{cm}^{-3} \mathrm{~s}^{-1}$ in one of the fingers in region $\mathrm{D}$. If the material is pure helium which is at most singly ionized, so that $n_{\mathrm{He} \mathrm{I}}=n_{e}$, then this emissivity implies $n_{e} \sim 10^{3} \mathrm{~cm}^{-3}$. This density, which is already a factor of 4 higher than the inferred density at the interface, is a lower limit. The strong [O I] emission indicates that the $\mathrm{He}$ is mostly neutral, so the total density may be much higher. Thus, even at this location, where the interface is only marginally unstable, the effects of cooling have lead to what is probably at least an order of magnitude enhancement in the density. Most of the material along the interface has probably run down the necks of the R-T fingers and is concentrated in these structures.

\section{DISCUSSION}

We have provided a quantitative physical basis for understanding the properties of the filaments in the Crab Nebula in terms of magnetic R-T instabilities between the synchrotron nebula and ejecta from the explosion. There are many interesting implications of this work for an overall understanding of the Crab, two of which are especially noteworthy.

\subsection{Effect of Field Strength on Ionization Structure}

Initially, we have found that the ionization structure of the R-T fingers varies with $\rho / \rho_{\text {crit }}$ in a systematic fashion. The 


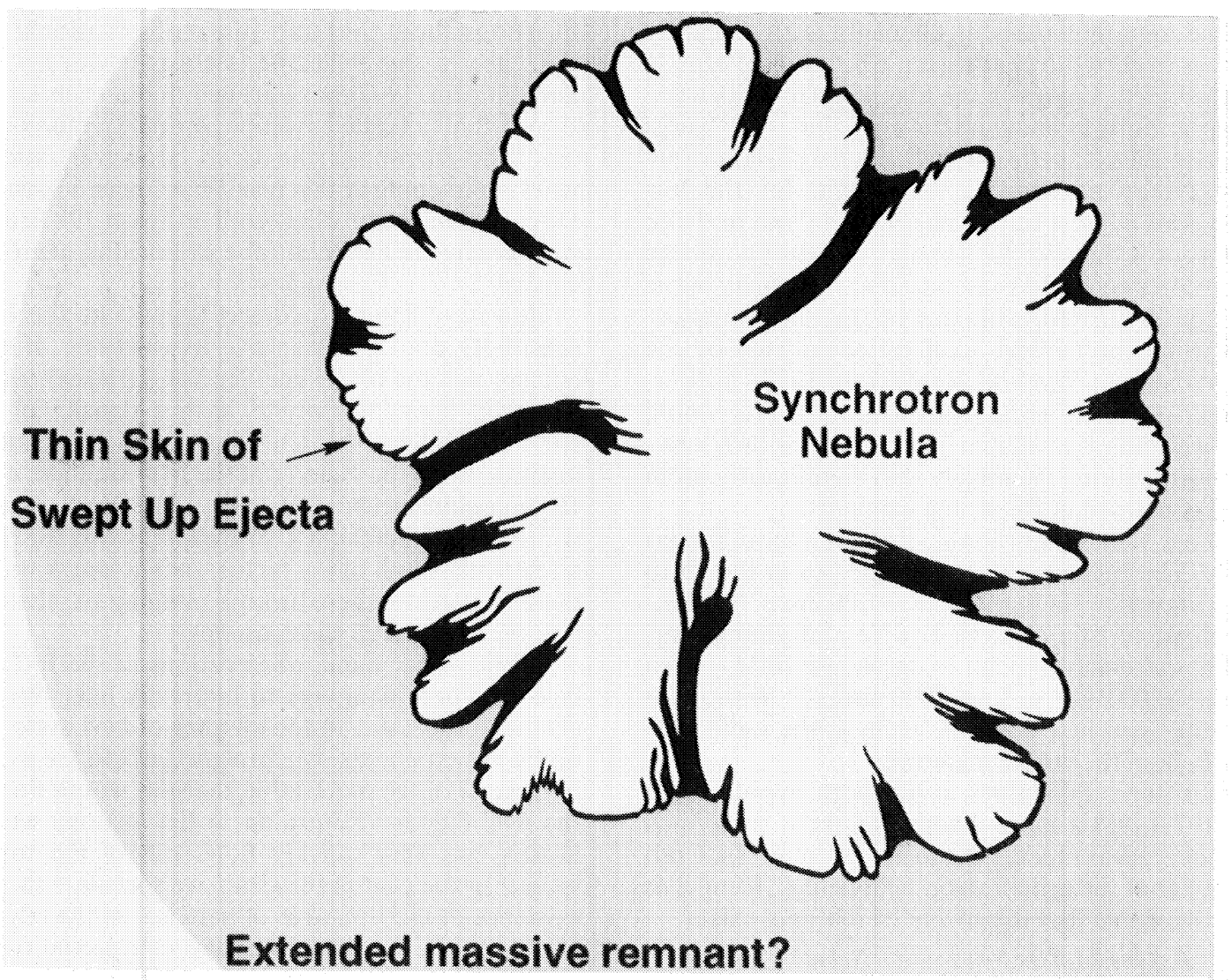

FIG. 8. - Schematic diagram showing a sketch of the hierarchical structure of filaments in the Crab Nebula. Structures at the smallest scales, seen in WFPC2 images, are quantitatively shown to be the result of R-T instabilities at the interface between the synchrotron nebula and swept-up thermal ejecta. Larger scale structures are likely the result of similar instabilities.

calculations in Figure 3 show that a strong magnetic field prevents the formation of the extended region of intermediate density material which surrounds the dense fingers in the nonmagnetic case. This difference is apparent in the observed structure of the Crab filaments. In features which are magnetically dominated (e.g., regions $\mathrm{C}$ and $\mathrm{D}$ ), high-ionization envelopes are generally not seen to surround the R-T fingers. Even in the presence of a UV radiation field which could lead to an evaporative filament profile, these filaments are magnetically confined and do not develop stratified ionization structures (at least at the resolution of the HST). As the dominance of the magnetic field declines, however, a stratified "core-halo" ionization structure does become apparent (e.g., regions E-H). The most extreme case (region I) consists of numerous lowionization knots embedded within a high-ionization envelope (cf. the structure in Fig. $3 b$ ).

This relationship between significance of the magnetic field and ionization structure confirms the suggestion (Hester et al. 1990) that variations in the degree of magnetic confinement in filaments significantly affects the relative prominence of lines formed in the $\mathrm{H}^{0}, \mathrm{H}^{+} \rightarrow \mathrm{H}^{0}$, and $\mathrm{H}^{+}$zones. This will likely have significant implications for the interpretation of spectra of $\mathrm{Crab}$ filaments. For example, it is worth noting that a number of knots which were identified by MacAlpine et al. (1989) on the basis of their peculiar velocities and peculiar spectra are clearly the dense heads of particularly long R-T fingers protruding into the nebula. This and other implications of the
WFPC2 data for the ionization structure of Crab filaments will be discussed in a subsequent paper.

\subsection{Origin of the Crab Filaments \\ 4.2.1. Missing Mass and Energy?}

Our findings concerning the character of R-T instabilities in the Crab may be relevant to a long-standing question about strength of the magnetic field satisfy the theoretical relationship between these quantities derived from the linear theory of magnetic R-T instabilities. This agreement gives strong evidence that the instability is in fact responsible for the observed structure, placing the earlier morphological arguments on solid, quantitative ground.

Numerical simulations of magnetic $\mathrm{R}-\mathrm{T}$ instabilities provide additional insight into the physical conditions in the Crab. These simulations show that the morphology of $\mathrm{R}-\mathrm{T}$ fingers in the nonlinear regime is a strong function of the relative importance of buoyancy forces and magnetic tension at the interface. When magnetic tension dominates, the interface is stable. If magnetic tension is unimportant, a classical nonmagnetic $\mathbf{R}-\mathbf{T}$ instability ensues. For intermediate cases, the magnetic field more or less suppresses secondary K-H instabilities and confines the dense fluid to long, sharply bounded fingers. This continuum of morphologies agrees remarkably well with the range of morphologies observed in the Crab. If the field strength at the boundary of the synchrotron nebula is fairly 
the remnant. The apparent mass of filaments is significantly less than the mass expected to have been ejected in a Type II SN. Similarly, the kinetic energy of the filaments (roughly a few times $10^{49}$ ergs) is only a small fraction of the $\sim 10^{51}$ ergs expected to be released as kinetic energy by the explosion.

In principle, it may be possible to account for the low inferred mass by hiding most of the mass of the Crab in dense neutral filaments cores. The WFPC2 images certainly show the existence of such cores, as well as call into question many of the assumptions made in current estimates of the filament mass. However, even using the modest acceleration implied by the proper motion data from Wyckoff \& Murray (1977), accelerating a total filament mass greatly in excess of $2 M_{\odot}$ would require more power than is available from the spin-down luminosity of the pulsar, making it unlikely that significantly more mass than this is contained in the visible filaments.

The low observed velocities probably present a more fundamental difficulty. The highest observed velocities in the Crab filaments are significantly less than the $\sim 10,000 \mathrm{~km} \mathrm{~s}^{-1}$ expansion velocities characteristic of Type II SNe and young remnants; it would be extremely difficult to decelerate $10 M_{\odot}$ or so of material from $10,000 \mathrm{~km} \mathrm{~s}^{-1}$ to $2000 \mathrm{~km} \mathrm{~s}^{-1}$ without it being apparent that you had done so, while at the same time accelerating the bulk of the visible material.

As a way out of these difficulties, it has been hypothesized (e.g., Chevalier 1977) that a more massive remnant surrounds the $\mathrm{Crab}$, and most recent models of the evolution of the Crab and the confinement of the synchrotron nebula (e.g., Kennel \& Coroniti 1984a, b) assume that this extended remnant exists.

$$
\begin{gathered}
\text { 4.2.2. Expansion of the Synchrotron Nebula } \\
\text { through a Massive Remnant? }
\end{gathered}
$$

If most of the ejecta from the Crab resides in an extended envelope of freely expanding ejecta, then the synchrotron nebula is expanding through this envelope. The expanding synchrotron nebula would then be acting as a snowplow, sweeping up and compressing material which would cool and continuously add to the skin on the surface of the synchrotron nebula. (See Figs. 2 and 8.)

On the basis of the work presented here, there are two possible fates for the swept-up material. The first is that eventually enough mass collects for the surface to become R-T unstable; $\mathrm{R}-\mathrm{T}$ fingers form, and the swept-up material flows into these fingers. The second possibility is that the swept-up material flows "downhill" across the surface of the bubble until it "drains" into existing R-T fingers. In either case the magnetic R-T instabilities in the Crab serve to concentrate swept-up ejecta into dense radially aligned structures, allowing the buoyant synchrotron nebula to expand outward through the ejecta.

Comparison of WFPC2 images with the linear theory and numerical simulations of $\mathrm{R}-\mathrm{T}$ instabilities lead us to make a strong claim that much and probably most of the structure of the filaments arises from a R-T unstable interface between the synchrotron nebula and swept-up ejecta. If a massive remnant surrounds the $\mathrm{Crab}$, then formation of filaments in the $\mathrm{Crab}$ is an ongoing process. If this is the case, then the currently observed filament mass is just a measure of how much of the ejecta has been swept up by the expanding synchrotron nebula to date.

\subsubsection{Can an Extended Remnant Be Inferred?}

It should be noted that while hypothesizing an extended massive envelope may help to answer a number of pressing questions about the Crab, direct observational evidence for the existence of this envelope is elusive. Murdin \& Clark (1981) and Murdin (1995) have argued that a deep UK Schmidt plate shows that an $\mathrm{H} \alpha$ envelope with an emission measure of 7 $\mathrm{cm}^{-6} \mathrm{pc}$ surrounds the Crab. However, attempts by other workers to confirm this result have been unsuccessful (see, e.g., Davidson \& Fesen 1985; Gull \& Fesen 1982), and a number of workers in the field continue to find the Murdin claim inconclusive.

However, failure to detect a halo at the limit of current sensitivity does not disprove its existence. If spread over the current volume of the nebula, $2 M_{\odot}$ (the estimated mass of the observed filaments) would have a density of $\sim 6 \times 10^{-24} \mathrm{~g}$ $\mathrm{cm}^{-3}$. If material of that density and the same average composition filled the vicinity of the Murdin halo, it would have an $\mathrm{H} \alpha$ emission measure of only $\sim 0.5 \mathrm{~cm}^{-6} \mathrm{pc}$ in a line with a width of over $5000 \mathrm{~km} \mathrm{~s}^{-1}$. Even allowing for the possibility of a relatively small filling factor, such a halo would be extremely hard to detect either through imaging or, thanks to the large line width, through spectroscopy.

On the other hand, observations of the R-T unstable interface may provide a way to indirectly infer the existence of an extended remnant. In the absence of new material being swept up by the expanding synchrotron bubble, we would expect that in time all of the material in the skin would flow downhill into the R-T fingers. (Returning to the analogy with solar prominences, gas very quickly flows out of the tops of magnetic loops.) In the case of the Crab, we speculate that the continued existence of the thin skin of material at the edge of the bubble might require continued "infall" of material onto the bubble's surface. If the time required to "drain" the skin into R-T fingers is shorter than the age of the remnant, then the amount of material at this surface would be the result of a balance between the rate at which material is swept up and the rate at which it drains into the fingers. We emphasize that current models do not allow us to make a strong claim regarding the existence of an extended massive remnant, but foresee that more realistic future simulations might be useful in this regard.

We note in passing that the existence of an extended massive remnant would have implications for other ideas about the $\mathrm{Crab}$. In particular, suggestions that the structure of the Crab may be influenced by circumstellar or interstellar matter would probably not be consistent with the existence of an extended envelope of ejecta carrying most of the mass and kinetic energy of the explosion. This outer ejecta would have interacted violently and visibly with any significant concentration of mass that it encountered. Examples of such ideas include the suggestion by Fesen, Martin, \& Shull (1992) regarding the origin of the synchrotron bays, or the suggestion that strong hydrogen emission in the northern part of the Crab (MacAlpine et al. 1989) may be due to swept-up interstellar gas.

\section{SUMMARY}

The Crab synchrotron nebula appears to be covered by a thin "skin" of swept-up thermal ejecta which is being accelerated by the pressure of the synchrotron plasma. It is clear that such an interface, with a light relativistic plasma accelerating denser thermal material, should be R-T unstable; WFPC2 images give strong morphological evidence that this instability is of paramount importance in determining the current structure of the remnant. Here we show that independent measurements of the apparent wavelength of the instability, the density of material at the interface, the rate of acceleration, and the 
uniform within the Crab, then the relative importance of buoyancy and Lorentz forces can be parameterized by the ratio of the density of thermal material at the interface to a critical density. The brightness and ionization structure of the emission give secondary indications of the density and are seen to vary systematically with the morphology of $R-T$ fingers in the way expected.

There are a number of caveats concerning the application of the currently available numerical simulations to the Crab. The most significant of these is that the current simulations neglect the effects of cooling. In the Crab, radiative cooling of material in the fingers should lead to a siphon effect, which will speed up the rate at which material at the interface collects in the R-T fingers. Other questions not addressed here include the organization of filaments into a lattice-like structure over the face of the nebula. We hope to address these and other issues with future simulations tailored specifically for conditions within the Crab.

These caveats notwithstanding, we conclude that magnetic $\mathrm{R}-\mathrm{T}$ instabilities between the synchrotron nebula and swept-up ejecta provide a consistent and quantitative framework within which to interpret the structure of the Crab Nebula. This leads to a number of interesting implications and speculations. The ionization structure of the R-T fingers varies systematically as a function of the relative importance of the magnetic field. This confirms an earlier suggestion that the degree of magnetic confinement should have a significant effect on filament spectra. It also seems likely that the hierarchy of filamentary structure in the Crab is due to the growth of multimode instabilities as the synchrotron nebula expanded through a region of originally more uniformly distributed ejecta.

Finally, we note that if much of the mass of the Crab exists in an extended remnant surrounding the currently visible nebula, as has been suggested elsewhere and often assumed in theoretical discussions of the nebula, then formation of filaments by $\mathrm{R}-\mathrm{T}$ instabilities is an ongoing process.

J. M. S. would like to acknowledge support from a faculty research grant from the University of Maryland. This work was supported by NASA contract NAS 7-1260 to the WFPC2 IDT. This work was supported at ASU by NASA/JPL contracts 959289 and 959329.

\section{REFERENCES}

Bandiera, R, Pacini, F \& Salvati, M 1983, A\&A, 126, 7

Bietenholtz, M. F., et al. 1991, ApJ, 373, L59

Chandrasekhar, S. 1961, Hydrodynamic and Hydromagnetic Stability (Oxford: Oxford Univ. Press)

Chevalier, R. A. 1977, in Supernovae, ed. D. N. Schramm (Dordrecht: Reidel), 53

Chevalier, R. A., \& Gull, T. R. 1975, ApJ, 200, 399

Clark, D. H., Murdin, P., Wood, R., Gilmozzi, R. Danziger, J., \& Furr, A. W. 1983, MNRAS, 204, 415

Davidson, K., \& Fesen, R. A. 1985, ARA\&A, 23, 119

Fesen, R. A \& Kirshner, R. P. 1982, ApJ, 258,

Fesen, R. A., Martin, C. L., \& Shull, J. M. 1992, ApJ, 399, 599

Gardner, C. L., Glimm, J., McBryan, O., Menikoff, R., Sharp, D. H., \& Zhang, Q. 1988, Phys. Fluids, 31, 447

Graham, J. R., Wright, G. S., \& Longmore, A. J. 1990, ApJ, 352, 172

Gull, T. R., \& Fesen, R. A. 1982, ApJ, 260, L75

Hester, J. J., Graham, J. R., Beichman, C. A., \& Gautier, T. N., III. 1990, ApJ, 357,539
Hester, J. J., Scowen, P A. \& Gallagher, J S. 1994, BAAS, 26, 95

Hester, J. J., et al. 1995a, ApJ, 448, 240

. 1995b, in preparation

Jun, B.-I., Norman, M. L., \& Stone, J. M. 1995, ApJ, 453, in press (JNS95)

Kennel, C. F., \& Coroniti, F. V. 1984a, ApJ, 283, 694 $1984 b$, ApJ, 283, 710

Lawrence, S. S., MacAlpine, G. M., Uomoto, A., Woodgate, B. E., Brown, L. W., Oliversen, R. J., Lowenthal, J. D., \& Liu, C. 1995, AJ, 109, 2635 Low, B. C. 1990, ARA\&A, 28, 491

MacAlpine, G. M., McGaugh, S. S., Mazzarella, J. M., \& Uomoto, A. 1989, ApJ, 342, 364

Michel, F. C., Scowen, P. A., Dufour, R. J., \& Hester, J. J. 1991, ApJ, 368, 463

Murdin, P. 1995, MNRAS, 269, 89

Murdin, P., \& Clark, D. H. 1981, Nature, 294, 543

Rees, M. J., \& Gunn, J. E. 1974, MNRAS, 167, 1

Trimble, V. L. 1968, AJ, 73, 535

1982, Rev. Mod. Phys., 54, 1183

Wyckoff, S., \& Murray, C. A. 1977, MNRAS, 180, 717 